\title{
An Active Learning Environment to Improve First-Year Mechanical Engi- neering Retention Rates and Software Skills
}

\section{Benjamin B. Wheatley, Colarado State University}

Benjamin Brandt Wheatley was awarded a B.Sc. degree in Engineering from Trinity College (Hartford, CT, USA) in 2011. He spent one year in industry at a biomedical device company before returning to graduate school. He is a doctoral candidate in the Department of Mechanical Engineering at Colorado State University (Fort Collins, CO, USA). His engineering education areas of interest include cultural competency, active learning approaches as they relate to software skills, and how "soft skills" project to success as engineers. His areas of technical research include finite element analysis and skeletal muscle mechanics.

\section{Dr. Tammy Lynn Haut Donahue, Colorado State University}

Tammy Haut Donahue joined the faculty at Colorado State University (CSU) in December of 2011. She came to CSU after spending eleven years in Mechanical Engineering at Michigan Technological University. Her PhD was in Biomedical Engineering from the University of California at Davis where she earned the Allen Marr Award for distinguished dissertation in Biomedical Engineering in 2000. She is an Associate Editor for the Journal of Biomechanical Engineering and an Editorial Consultant for the Journal of Biomechanics. She is Chair of the Orthopaedic Research Society Meniscus Section, and is a member of the Bioengineering Executive Committee for the American Society of Mechanical Engineers. Dr. Haut Donahue's research includes analytical and experimental biomechanics of the musculoskeletal system with ongoing research in orthopedic biomechanics and post-traumatic osteoarthritis. An emphasis is put on prevention, treatment, and repair of injuries to the soft tissue structures of the knee, focusing primarily on the meniscus. With over $\$ 15$ million in funding from Whitaker Foundation, CDMRP, NIH, NSF, as well as industrial sponsorship her research program has had more than 60 mentees and has national collaborations with Michigan State and Mayo Clinic as well as international collaborations with Trinity College Dublin and Queens University Belfast. Dr. Haut Donahue has more than 65 peer-reviewed publications and is current Associate Department Head for Undergraduate Studies for the Mechanical Engineering Department at CSU. Dr. Haut Donahue was awarded the Ferdinand P. Beer and E. Russell Johnson Jr. Outstanding New Mechanics Educator Award from the American Society of Engineering Education for exceptional contributions to mechanics education. Dr. Haut Donahue is a fellow of the American Society of Mechanical Engineers.

\section{Dr. Kimberly Catton P.E., Colorado State University}

Professor of Practice Mechanical Engineering 


\title{
An Active Learning Environment to Improve First-Year Mechanical Engineering Retention Rates and Software Skills
}

\begin{abstract}
This work proposes a foundational change from traditional lecture to an active learning environment in the Colorado State University First-Year Introduction to Mechanical Engineering course of 145 students. The goal of this approach is to improve computational capabilities in Mechanical Engineering and long-term retention rates with a single broad emphasis. Major and minor changes were implemented in the course, from specific day to day in-class activities to the addition of laboratory sessions to replace traditional classroom lecture. These laboratories of no more than fifteen students were delivered by Learning Assistants, which were upper-level undergraduate peer educators. To evaluate proficiency, a MATLAB post-test was delivered to students who were instructed through lecture only ("Lecture") and those who were instructed with the above changes ("Active"). A survey was also provided upon completion of the course to the Active group for student reflection on their perceived software capability and the usefulness of approaches. Post-test results suggest that the Active group was more proficient in MATLAB than the Lecture group. Survey results suggest that the Active group recognize they had not achieved expert use of the software but that they were likely to use it throughout their careers and that all approaches were useful, in particular the use of Learning Assistants. Future longterm retention statistics will shed light on the possible effectiveness of this approach, which are currently unavailable.
\end{abstract}

\section{Introduction}

Colorado State University has a total student enrollment in excess of 33,000. As a land grant university, the historic mission of the institution is to provide students with an education in practical fields such as agriculture and engineering. The College of Engineering has a growing student cohort, with an increase from 450 first-year students Fall 2010 to 600 students Fall 2015 [1]. However, persistence and graduation rates have remained fairly steady over the last fifteen years. The current six year persistence rate within the college is only $\sim 45 \%$ and the six year graduation rate within the college is similar at $\sim 43 \%$. Many students do not remain within the college for even a full year, as the second fall persistence rate is only $70-75 \%$ [1]. These data show a significant portion of enrolled first-year engineering students do not remain within the program long enough to be exposed to foundational engineering content, which starts in the sophomore year with engineering specific courses. A current goal of the college is to improve these retention statistics.

Additionally, many students do not develop the necessary software skills required to use computational tools such as MATLAB, which are integral to success in the curriculum. Students who do not develop these skills during introductory coursework must "catch up" in later courses, where the technical content is more challenging. We hypothesize this can lead to unpreparedness for challenging content or careers as an engineer and can negatively impact academic standing, leading to decreased retention. Thus, the goals of this work were to 1) improve retention rates for first-year engineering students, specifically mechanical engineering, and 2) improve computational and software skills of first-year students, specifically MATLAB and Microsoft Excel. 
MATLAB is a common computational package which can be used for a broad range of engineering problems throughout a curriculum [2]. However, learning Excel and MATLAB through lecture is challenging, as these tools are best understood through utilization, not observation [3]. MATLAB and other computational tools are often taught in classrooms with computational equipment, however this is can be a challenge with a large classroom [4]. Some have utilized computer based tutorials which students can complete on their own time [5], while others implemented a large scale deployment of personal computers equipped with MATLAB and other software [6]. Additionally, the use of peer-educators can be an effective approach to facilitating MATLAB development [7]. Thus, we have chosen to employ an approach which utilizes an active environment to learn MATLAB and other introductory content through the use of laboratory sessions and peer-educators, in this case the Learning Assistant model [8]. Similar to previous approaches, we have utilized classroom lectures, hands on in-class activities, and laboratory sessions [9].

The Introduction to Mechanical Engineering Course (MECH 103) was developed to provide students with an overview of the mechanical engineering discipline and as an introduction to the computational packages MATALB [10] and Microsoft Excel. The course consists of between 140 and 250 first-year students and was previously delivered using traditional lecture. While this approach was most efficient for a single instructor due to the enrollment size, this resulted in a static learning environment for a course which should excite students about mechanical engineering and provide foundational technical skills. The overall approach to this work was to thus create an active environment for students within the course, which had an enrollment of 145 students for the Fall 2016 semester. The rationale to this approach was that by providing students with hands-on experiences working with mechanical engineering problems and computational software, the understanding of course content will improve $[11,12]$ whereby improving retention [13]. While some immediate test and survey data were acquired and are shown in this work, it is important to note that the true impact on retention is not currently recognizable and will require future analysis.

\section{In-Class Sessions}

Class sessions were varied throughout the semester and the week, as they typically included lectured course material, guest lectures or panels, and activities. The course met Monday, Wednesday, and Friday from 9-9:50 AM in a large lecture hall with individual stadium seating. Friday lecture was often cancelled and this time was spent in weekly laboratory sessions instead, which are outlined in the next section. Monday class time was assigned to covering course content through lecture, teamwork activities, and in class problems. The content of the course included general introductory material such as teamwork, communication, and design, commonly used units and unit conversions, mathematical models and systems, and an introduction to Microsoft Excel and MATLAB.

Active engagement in the class included a teamwork design problem, requiring students to break into groups of three. Due to the theater seating layout of the classroom, groups of four or more made successful teamwork and communication difficult. Each group of students were provided one piece of 8.5 " x 11 " blank printer paper, one paperclip, and two pieces of scotch tape. The design problem was simple: build the tallest free standing structure possible using only the given materials. This was an inexpensive and simple approach to teamwork design activity. In place of 
a lecture or even a discussion on how to use design techniques for a simple problem such as this, students were able to actively engage in this process despite the difficulties of class size and layout. While students typically have an excellent understanding of units such as a pound (lb), their physical understanding of units such as a Joule or Watt are less developed within the context of everyday life. To provide students with a meaningful representation of energy (Joule) and power (Watt), they were provided a common object - in this case a softball - and asked to calculate how high they would have to raise the object to exert one Joule of energy - in this case roughly a foot and a half. While simple and inexpensive, this activity provided students with useful knowledge they can apply without a calculator and helps them relate coursework to the real world. For example, if they can place a Joule into real-world context, they could then answer the question "Can I launch a rocket into space using a thousand Joules?".

Wednesday lecture sessions were commonly used for guest lecturers and panels. These class sessions included the College of Engineering Dean, faculty members and graduate students in mechanical engineering, industry panelists, entrepreneurs and small business owners, and an interactive teamwork theatre troupe. The goal of these sessions was to provide students with a broad overview of different disciplines within mechanical engineering and what skills are necessary to succeed in various professional roles. While emphasizing an active learning environment is inherently difficult with each and every guest, student engagement was addressed by delivering variability in all of the presentations and strongly encouraging students to ask questions. For example, the theater troupe was an interactive experience where students were able to act as a team member within a group that mocked to show a diverse team struggling with communication. This session involved humor, discussion, and lively responses from students in place of a traditional static lecture.

\section{Laboratory Sessions}

In place of Friday lecture, students were asked to attend laboratory sessions for one hour [14,3]. A total of eleven sessions were provided throughout the week to accommodate all schedules. Sessions included one instructor, 13-16 students, and were held in laboratories with individual workstations with Microsoft Excel and MATLAB software. Laboratory instructors included a Graduate Teaching Fellow and Undergraduate Learning Assistants (LAs).

Laboratory sessions involved a short ( $<5$ minutes) lecture briefly reviewing content from class before students began working on assigned problems. These problems implemented course content such as the use of Excel or MATLAB to analyze and display data through real-world applications. An example of utilizing MATLAB to simulate rolling a die is provided:

You will develop an indefinite loop (while) which will simulate rolling a die until a 6 is rolled. Use the randi function to return one value between 1 and 6 at a time, and repeat this within a while loop with the condition of the value being less than 6. How many runs did it take before the first 6 was rolled?

Perform this same simulation 1,000 times and determine the average number of rolls before the first 6 is rolled. Plot the number of rolls it takes until a 6 is observed (1,000 data points) using the histogram function in MATLAB.

This problem required students to use both an indefinite (while) and definite (for) loop, nest the loops appropriately, and visually display generated data. It is important to note that while the 
general structure of the necessary code would likely be similar for all students, there is no single "right answer". This is often the case for professional engineers. These laboratory sessions allow students to explore the software and develop their own problem solving skills in place of observing an instructor solve a problem in a single manner during a lecture. Developing a link to real-world problems with these assignments helps students interpret products such as MATLAB as a useful tool and not only a necessary requirement for academic success. Learning to use Excel and MATLAB effectively early in their undergraduate career will hopefully facilitate further implementation throughout the engineering curriculum, which may help with retention. However, if students feel burdened by learning what they interpret as a complex programming language such as MATLAB, it could certainly have an opposite effect.

Specific content covered in these sessions include the following for Microsoft Excel: cell referencing, data analysis, visual plotting, curve fitting, and statistics. For MATLAB: variable types, matrix mathematics, for and while loops, conditional operators, if statements, importing and exporting with Excel, and functions. Additionally, an introduction to the facilities and engineering resources, units, communication, and teamwork were regularly included in assignments throughout the semester. As an example, students were asked to design their own unit and orally present to their peers the conversion from their unit to a commonly used SI equivalent, and describe a scenario in which one would use their unit in place of another. A realworld example of this question is a furlong, which is a measurement of length commonly used in horse racing.

\section{Implementation of Learning Assistants}

Three undergraduate Learning Assistants (LAs) [8] were selected and employed to deliver weekly laboratory sessions. Each LA was assigned three sessions of active teaching per week within the laboratory at one hour per session. LAs also met with the Faculty Instructor and Graduate Teaching Fellow on a weekly basis to discuss and review course content, student success, and general observations. LAs were also provided weekly pedagogical training opportunities through a teaching and learning institute. The three Learning Assistants selected for this course (two male, one female) were from three different academic years (sophomore, junior, and senior), and thus provided diverse viewpoints on the mechanical engineering program and course content.

The major benefit of the LA program is consistent student interaction with the LAs, in this case within the laboratory setting. Each LA was given the same laboratory time slots with the same students, allowing students to develop personal relationships with an instructor figure despite the large class size. One goal from this work was the provide the first-year students with an environment in which they feel comfortable making mistakes, asking questions, and discussing material outside of a lecture hall with 150 people or a one-on-one office hours session with faculty. This was partially achieved because the LAs were tasked with providing feedback - not evaluations - in a low stakes environment to students, which facilitated the development of technical skills over simply receiving a good grade.

One tertiary benefit of the Learning Assistant program is the development of important skills by the LAs themselves. Specifically, the LAs learned the course material at an instructor level, which in the case of Excel and MATLAB is a career-long skill for engineers. In fact, one of the 
LAs had no specific MATLAB training prior to the program and was excited with the usefulness of the software. LAs also improved their communication and pedagogical abilities by leading sessions, working with students one-on-one, and designing laboratory content themselves. This was observed during the weekly meetings with the LAs, where difficult scenarios such as unprofessional students and LA comfort with delivering content were discussed.

\section{Evaluative Methods}

To evaluate the effectiveness of the course changes on technical content, two groups of students completed a MATLAB post-test after completion of the course. The first group completed MECH 103 prior to the above implementations ("Lecture") and the second group was taught the course as outlined above ("Active"). The post-test consisted of six questions (see Appendix), each covering separate components of MATLAB content. An identical test was given to each group of students, thus direct comparisons can be made. This post-test was delivered in the first week of the Mechanical Engineering course immediately following MECH 103 (Mechanical Engineering Problem Solving) which has a heavy MATLAB component. Future work to validate this post-test and potentially develop a MATLAB concept inventory would benefit this work. The post-test was developed to cover a wide range of introductory MATLAB content, ranging from matrices to loops and conditional statements, and included MATLAB specific questions as well as those which required general programming knowledge (see Appendix).

Students in the Active group were also provided with a survey to reflect on their MATLAB and Excel capabilities and which portions of the course they found to be most helpful. The survey was developed by a group of faculty members and graduate teaching fellows and was delivered using a Likert scale. The survey was designed to provide students with an opportunity to reflect upon two vital topics related to the course content: 1) student perceived capability of learning and utilizing MATLAB and Excel, and 2) student perceived usefulness of the approaches enacted in the course. Specifically, the capability questions included those on how proficient the students felt in the software, how comfortable they felt utilizing the software, and how likely they were to use MATLAB and Excel in the future. From an approach standpoint, students were asked to specify if they felt the in class activities, laboratory sessions, and Learning Assistants were helpful. All questions were based on a five-point scale, with one being lowest capability or usefulness, and five being most capability or usefulness. The survey was designed in this fashion to provide students with specificity within each topic, while also collecting data on the overall perceived effectiveness of the enacted changes. It should also be noted that this survey was delivered as a portion of a general survey focusing on retention rates within the College of Engineering. While a validation and/or reliability study was not possible due to time constraints, this could greatly improve future impact of this work.

The survey provided in this work is also closely linked to Utility Value, which is a subcategory of Expectancy-Value Theory [15,16]. In short, the Expectancy-Value Theory suggests that the decision-making process and success of students is largely impacted by two factors: their expected success for some task and the value associated to that task. Utility Value takes this a step further to specifically explore the expectancy and value associated with tasks which are perceived as useful (in comparison to tasks which are primarily for enjoyment or self-identity). The results of this survey will thus shed light onto the perceived expectancy of success and value of the course. 


\section{Findings}

Post-test results (Table 1) show drastic increases in correct responses from the Lecture to the Active group. A Chi-Square test was performed for each question with the null hypothesis of no relationship between group and question correctness. For each question except "Matrix Indexing", the null hypothesis was rejected with very low $p$-values $(\mathrm{p} \leq 0.001)$, strongly supporting the notion that the Active group performed better on the post-test. In some cases, students in the Active group were nearly six times more likely to answer questions correctly than the Lecture group. The Matrix Indexing problem had low percent correct for each group and neither group were more likely to answer correctly $(\mathrm{p}=0.53)$.

While this is strong evidence suggesting the course changes outlined above are effective in facilitating the development of MATLAB skills, it should be noted there are likely other factors which contribute to these observed differences. Firstly, differences in content emphasis may play a role, as the Lecture and Active groups were taught by different instructors. The Lecture group also enrolled in introduction to mechanical engineering in the 2016 Spring semester and completed the post-test during the 2017 Fall semester. The Active group completed the course in the 2016 Fall semester and completed the post-test in the 2017 Spring semester. The time difference between these two groups (three months versus one month) could have led to increased retention in the Active group. However, these differences alone are unlikely to result in the number of correct responses increasing two to six fold. Additionally, it should be noted that the post-test utilized in this study could benefit greatly from a validation to further strengthen the findings.

Table 1. Percentage of students who responded correctly to post-test questions on MATLAB content for two groups: those taught the introduction to mechanical engineering course prior to implementation of active components ("Lecture") and those taught the course with active components ("Active"). A Chi-Square test showed statistical correlation between the Active group and correctly answering each question in comparison to the Lecture group (bold denotes $\mathrm{p} \leq 0.001$ which was achieved for each question, except "Matrix Indexing" which had $\mathrm{p}=0.53$ ).

\begin{tabular}{|c|c|c|c|c|c|c|c|c|c|}
\hline \multirow{2}{*}{$\begin{array}{c}\text { Question } \\
\text { Content }\end{array}$} & \multicolumn{4}{|c|}{$\begin{array}{c}\text { Array/Matrix } \\
\text { Multiplication }\end{array}$} & $\begin{array}{c}\text { Matrix } \\
\text { Indexing }\end{array}$ & Functions & $\begin{array}{c}\text { For } \\
\text { Loops }\end{array}$ & $\begin{array}{c}\text { If } \\
\text { Statements }\end{array}$ & $\begin{array}{c}\text { While } \\
\text { Loops }\end{array}$ \\
\cline { 2 - 9 } \\
\hline $\begin{array}{c}\text { Lecture } \\
(\mathrm{n}=73)\end{array}$ & $\mathbf{4 1 \%}$ & $\mathbf{3 7 \%}$ & $\mathbf{1 1 \%}$ & $\mathbf{8 \%}$ & $19 \%$ & $\mathbf{2 7 \%}$ & $\mathbf{1 9 \%}$ & $\mathbf{1 5 \%}$ & $\mathbf{3 6 \%}$ \\
\hline $\begin{array}{c}\text { Active } \\
(\mathrm{n}=97)\end{array}$ & $\mathbf{8 9 \%}$ & $\mathbf{8 4 \%}$ & $\mathbf{6 4 \%}$ & $\mathbf{3 3 \%}$ & $15 \%$ & $\mathbf{7 7 \%}$ & $\mathbf{5 4 \%}$ & $\mathbf{7 4 \%}$ & $\mathbf{6 2 \%}$ \\
\hline
\end{tabular}

From a capability standpoint (Figure 1), students felt they had obtained some level of both proficiency with software (median of 3 ) and comfort utilizing software (median of 4). However, there were few students who felt they had mastered these programs. This is not surprising given the complexity and capabilities of MATLAB itself, which can be used for data analysis, controls, and visualization, ranging from novice use to highly advanced scripting. Students did report they were likely to use MATLAB and Excel throughout their careers (median of 4). This mindset will likely benefit their continued development of these important computational engineering skills. Relating these findings to Expectancy-Value Theory and more specifically Utility Value, students associated high levels of value to MATLAB and Excel, yet their perceived success was 
somewhat lower. For first-year students, assigning a high level of value to a tool (MATLAB or Exel) which they have limited experience with was a success, as it will hopefully encourage them in the future to invest further in these programs. As Utility Value focuses on "useful" tasks (in contrast to those focusing on enjoyment, for example), it should be noted that this is only one portion of what students are likely motivated by. Additionally, MATLAB alone is a difficult software package to master in a short period solely based on its broad capabilities. Thus, there is little concern over students recognizing they have not reached expert level skill after only 5-6 weeks of study.

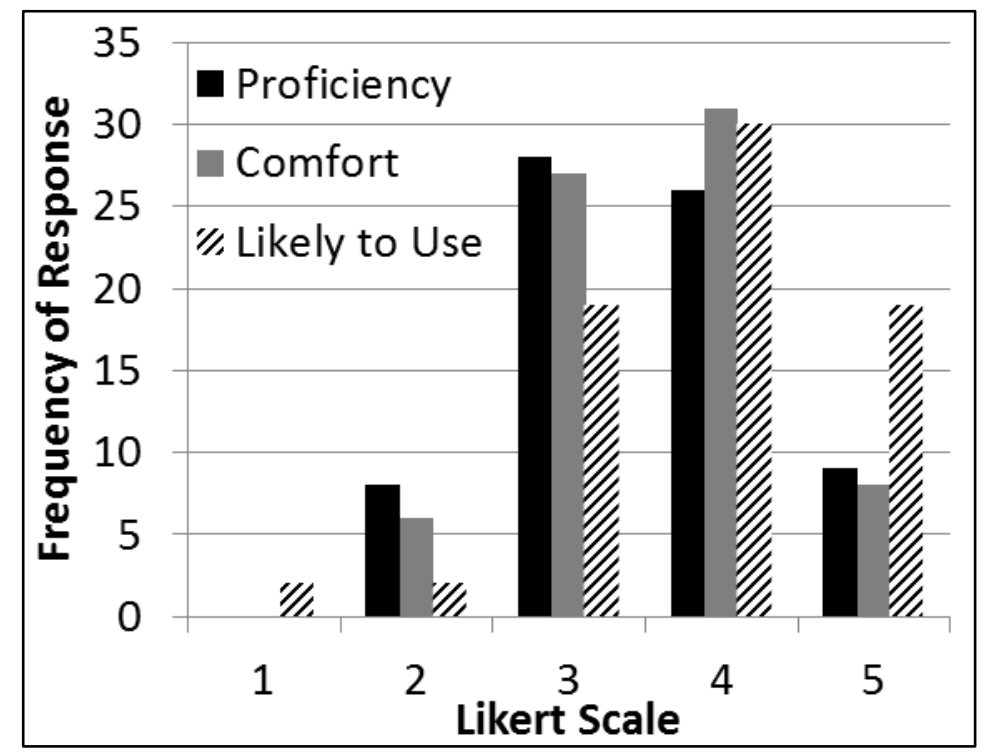

Figure 1. Student responses of their perceived capabilities utilizing MATLAB and Microsoft Excel measured through a Likert scale. "Proficiency" relates to how proficient students felt with software, "Comfort" relates to how comfortable students felt utilizing the software, and "Likely to use" relates to how likely students were to use MATLAB and Excel in their coursework or careers.

Finally, students were asked to specify how useful they felt the changes to the course were in terms of their educational experience (Figure 2). These changes were divided into three groups: laboratory sessions, in-class activities, and the use of Learning Assistants. The laboratory sessions were perceived as very helpful to students (median of 4), and while it appeared the class sessions were less helpful (median of 4) this was likely due to the difficulty of performing activities with $\sim 150$ students at once. This was supported by a nonparametric Mann-Whitney test ( $\mathrm{p}=0.0018$, Figure 3). Finally, students felt the Learning Assistants were the most helpful (median of 5). This was statistically significant in comparison to both in-class activities $(\mathrm{p}<0.0001)$ and laboratory sessions $(\mathrm{p}=0.047)$ as evaluated with a nonparametric Mann-Whitney test (Figure 3). This is likely the result of multiple factors, including employing Learning Assistants which not only understand the content but are passionate about education and the use of the LA model to provide beneficial feedback and not evaluative grades. 


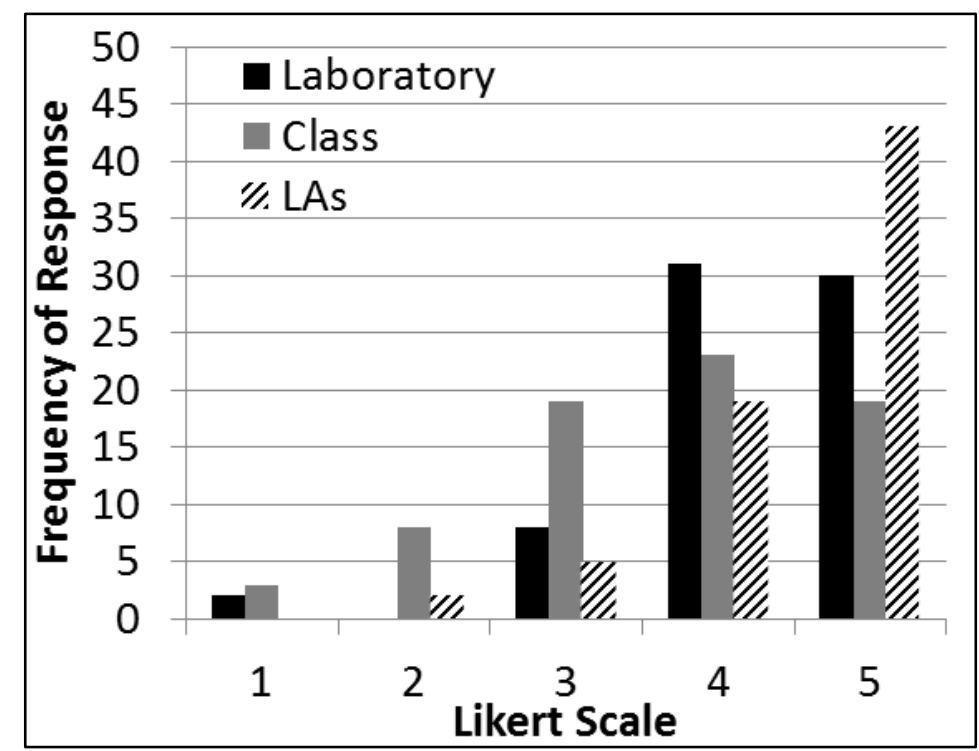

Figure 2. Student responses of the perceived effectiveness of various changes to the introduction to mechanical engineering course measured through a Likert scale. "Laboratory" related to the helpfulness of laboratory sessions, "Class" relates to the helpfulness of activities within the classroom, and "LAs" relates to the helpfulness of the Learning Assistants.

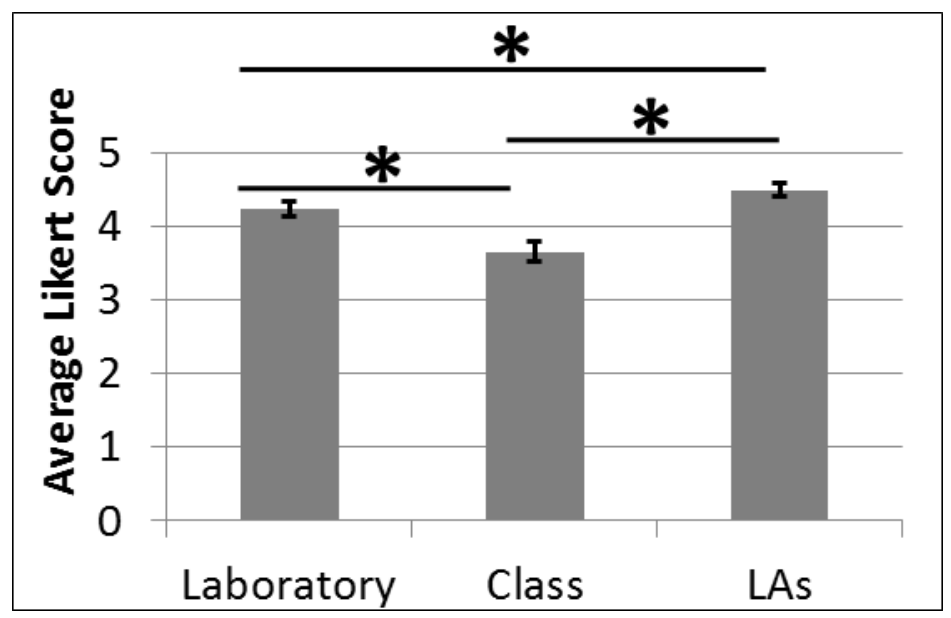

Figure 3. Statistical results of Active student group surveys for student perceived usefulness. A nonparametric Mann-Whitney test was used to compare Likert scale vales (* denotes significance at $\mathrm{p}<0.05)$. Data presented show mean and standard error of the mean bars.

\section{Conclusions and Future Work}

This work presented an approach to teaching computational proficiency and introductory content through a first-year introduction to mechanical engineering course. Specifically, the course was altered from a lecture-only environment to an active environment with laboratory sessions [10,3]. This allowed a large number of students to receive instructor support in smaller groups through Learning Assistants as a peer educator model and to work on computational content in a handson environment. Students showed increased computational proficiency, an appropriate sense of their technical capabilities, and felt the utilized approaches were effective. This work suggests the use of a broad approach to improve computational proficiency amongst first-year students. While it is unclear exactly how each constituent contributed to student success, the three 
approaches (laboratory sessions, in-class sessions, and the LA model) yielded favorable responses from students. This broad method is also likely to benefit students who learn through different teaching methods.

It should be noted the above methods and findings are only possible through investment by the department, college, and university to improve student learning. Enacting the outlined changes is simply not feasible with a single instructor, particularly in the case of a class of 250 students. To properly employ laboratory sessions and activities with such a large class, invested educators are absolutely critical. The Learning Assistant model, however, provides an effective approach to providing students with important interactions with peer educators. This peer learning model also provides a valuable opportunity for Learning Assistants themselves to develop useful technical and pedagogical skills [8].

While one overall goal of this work is to improve student retention, the effectiveness of this approach to retention rates remains unclear. These data will be available throughout the following years as the current group of undergraduates progress through the program.

Specifically, the second Fall semester (Fall 2017) will be of particular interest, as the persistence rate within the college is currently only $\sim 75 \%$ after one year [1]. While further changes to this and other courses throughout the curriculum could certainly improve student retention, they will thus make the impact of this work less clear. However, a concerted effort by the university, college, and department to improve retention rates can certainly be studied in the future with the 2016 first-year class as a focus.

\section{Appendix}

The post-test MATLAB questions are provided below:

1. What is the result of you execute the following statements in the MATLAB command window?
a. $A=[12 ; 34]$
b. $B=2 * A$
c. A.*B
d. $A * B$

2. Which command will return the corner elements of a 10-by-10 matrix A?
a. $\mathrm{A}([1$, end $],[1$, end $])$
b. $\mathrm{A}([1,1]$, [end,end] $)$
c. $A(\{[1,1],[1$, end $]$, [end, 1$]$, [end,end $]\})$
d. $\mathrm{A}(1$ :end, 1 :end $)$

3. Which command will create a plot of acceleration vs. time(i.e., a vector of time on the xaxis and a vector of acceleration on the y-axis)?

a. plot(time, acceleration) 
b. $\operatorname{plot}($ acceleration, time $)$

c. $\operatorname{plot}([$ time, acceleration] $)$

d. $\operatorname{plot}([$ acceleration, time $])$

4. What is the output of the executing the following MATLAB code?

$$
\begin{aligned}
& \text { clear; } \\
& \text { for } \mathrm{i}=1: 5 \\
& \text { for } \mathrm{j}=1: 5 \\
& M(i, j)=i+j ; \\
& \mathrm{M}(\mathrm{j}, \mathrm{i})=\mathrm{M}(\mathrm{i}, \mathrm{j}) \\
& \text { end } \\
& \text { end } \\
& \mathrm{M}(4,3)
\end{aligned}
$$

5. Use one if statement to rewrite the following nested if statements.

$$
\begin{aligned}
& \text { if } w<x \\
& \text { if } w>y \\
& \text { end } w=x * y
\end{aligned}
$$

6. Given the following MATLAB code, what is the value of $\mathrm{k}$ after executing?

$$
\begin{aligned}
& \mathrm{k}=0 \text {; } \\
& \text { while } \mathrm{k}^{\wedge} 0.5<\mathrm{k} \\
& \mathrm{k}=\mathrm{k}+1 \text {; } \\
& \text { end } \\
& \mathrm{k}
\end{aligned}
$$

\section{References}

[1] 2016, Colorado State University Retention Study.

[2] de Guzman, R., Vaccaro, J. C., Pesch, A. H., and Craig, K. C., 2016, "Freshman Engineering Problem Solving with MATLAB for All Disciplines," ASEE Annual Conference and Exposition, American Society for Engineering Education.

[3] Talbert, R., 2012, "Learning MATLAB in the Inverted Classroom," p. 25.883.125.883.19.

[4] Holbert, K. E., and Karady, G. G., 2009, "Strategies, Challenges and Prospects for Active Learning in the Computer-Based Classroom," IEEE Trans. Educ., 52(1), pp. 31-38.

[5] Daku, B. L. F., and Jeffrey, K. D., 2001, "Development of an interactive CDROM-based tutorial for teaching MATLAB," IEEE Trans. Educ., 44(2), p. 10 pp.

[6] Tront, J. G., 2007, "Facilitating Pedagogical Practices through a Large-Scale Tablet PC Deployment," Computer (Long. Beach. Calif)., 40(9), pp. 62-68.

[7] Reckinger, S. M., 2016, "Implementation and Evaluation of Different Types of Peer Learning Instruction in a MATLAB Programming Course," ASEE Annual Conference and Exposition. 
[8] Otero, V., Pollock, S., and Finkelstein, N., 2010, “A physics department's role in preparing physics teachers: The Colorado learning assistant model,” Am. J. Phys., 78(11), pp. 1218-1224.

[9] Hrynuk, J., Pennington, M., Illig, D., and Dempsey, J. P., 2008, "Freshman Engineering: An Introductory Computer Course Teaching Matlab And Labview," ASEE Annual Conference and Exposition, American Society for Engineering Education.

[10] Devens, P., 1999, "Matlab And Freshman Engineering," p. 4.378.1-4.378.6.

[11] Bonwell, C. C., and Eison, J. A., 1990, "Active Learning: Creating Excitement in the Classroom. 1991 ASHE-ERIC Higher Education Reports."

[12] Prince, M., 2004, "Does Active Learning Work? A Review of the Research," J. Eng. Educ., 93(3), pp. 223-231.

[13] Klingbeil, N., 2004, "Rethinking Engineering Mathematics Education: A Model For Increased Retention, Motivation, And Success In Engineering," p. 9.1068.1-9.1068.12.

[14] Foertsch, J., Moses, G., Strikwerda, J., and Litzkow, M., 2002, "Reversing the Lecture/Homework Paradigm Using eTEACH® Web-based Streaming Video Software," J. Eng. Educ., 91(3), pp. 267-274.

[15] Eccles, J., 1983, "Expectancies, values and academic behaviors.," Achievement and achievement motives: Psychological and sociological approaches, Freeman, San Francisco, CA, pp. 75-146.

[16] Wigfield, A., and Eccles, J. S., 2000, "Expectancy-Value Theory of Achievement Motivation," Contemp. Educ. Psychol., 25(1), pp. 68-81. 